\title{
Risk Simulation Analysis of Vehicle Moving on Curved Sections based on Driver Braking
}

\author{
Chi Zhang*, Dongjian Shao and Min Zhang
}

Key Laboratory for Special Area Highway Engineering of Ministry of Education, Chang'an University, Middle Section of Nan Erhuan Road, Xi'an, 710064, China

\begin{abstract}
In consideration of rainfall, over-speeding and road alignment, driver braking which caused the risk of vehicle moving on curved sections is analyzed synthetically. The simulation model of the dynamic characteristic of vehicle is established using Carsim, the critical adhesion coefficient and lateral acceleration are used as the evaluation index of vehicle moving risk, orthogonal design is used to reduce the test data, effect factors and the efficiency are analyzed based on the knowledge of mathematical statistics, the evaluation model about vehicle moving on curved sections is established using Matlab. The results show that speed and radius of horizontal curve have significant effects on the risk of driving, braking effect is moderate, super-elevation has few effect; when braking is moderate, even if with poor road alignment, fast speed and bad weather conditions, vehicle will not sideslip; when braking is heavy and over speeding, no matter the road alignment and the weather is good or bad, vehicle may sideslip. In addition, in the risk evaluation, meeting the requirement of adhesion coefficient, also meeting the requirements of lateral acceleration. The research results can provide guidance for risk analysis of vehicle moving on curved sections.
\end{abstract}

Keywords: Braking, carsim simulation, curve sections, risk analysis, road engineering, sideslip.

\section{INTRODUCTION}

Highway traffic accident causation including person, vehicle, road, environment, bad driving behavior, complex conditions of road alignment and infaust weather conditions are the part cause of traffic accidents. According to traffic accidents in China statistical yearbook [1], in 2005, the traffic accidents happened on curved sections accounted for $7.84 \%$ of total accidents, fatal accidents happened on curved sections accounted for $16.3 \%$ of total fatal accidents, Andrey and Yagar [2] studied different regional traffic accident history data, found that compared with sunny day, rainy day traffic accident rate increases by $70 \%$, according to the statistical material of ministry of public security traffic management bureau, in the first half of 2009, the fatal accidents which caused by over-speeding accounted for $13.8 \%$ of total fatal accidents, in addition, Zhoucheng Su [3] carried out simulation analysis on the turning braking condition, results show that when the speed is high, braking force had a great influence on the driving stability of vehicle. Therefore, considering the rainfall and over-speeding behavior, studying the braking behavior of driver on curved sections the risk of driving has great significance to improve the level of road safety.

Most traffic accidents have multiple influence factors, individual factors may not lead to the occurrence of traffic accidents, only when all these factors were involved in which can cause a traffic accident. Therefore, when analysis of the factors that affects the risk of driving, all factors should be taken into account. Besides, improving the quality of road alignment design in the road design stage can significantly improve the traffic safety level during operation, can not only reduce casualties, but also save a huge cost to improve road safety on dangerous sections, so the cost-benefit ratio on improving design in design stage is most satisfactory [4-7]. However, current design method based on design speed, processing complex of coupled vehicle systems into a rigid body simply, not considering the influence of the driver operation behavior, also not considering the influence of adjacent linear unit ahead, the habit of designed flat, vertical and cross section respectively can also lead to road alignment not match the driver and vehicle, in addition, also not considering the influence of weather.

The above problems as a starting point of this paper, the critical adhesion coefficient and lateral acceleration are used as the evaluation indices of vehicle moving risk, considering rainfall, over-speeding and road alignment, driver braking which caused the risk of vehicle moving on curved sections is analyzed synthetically. Firstly, analyzed the impact strength of driving risk with various factors, then analyzed driver braking which caused the risk of vehicle sideslip under the condition of rainfall and over-speeding, the evaluation model about the evaluation indices and influential factors is established, in addition, considering rainfall and overspeeding, driver braking which caused the risk of vehicle moving on curved sections is analyzed. 


\section{DRIVING RISK ANALYSIS AND RISK ASSESS- MENT INDICATORS}

\subsection{Driving Risk Analysis}

Vehicle with speed $V$ moving on curve sections with radius $R$ will be affected by the effect of centrifugal force, when the road cannot offer enough surface adhesion to resist centrifugal force, vehicle will produce sideslip dangerous working condition.

When the driver takes braking behavior, the longitudinal force of each tire has changed, load moving through tire caused tire vertical load changed. If only considering the effect of longitudinal force, when the lateral force of front and rear wheels is constant and the braking force of front wheels are larger than rear wheel, sideslip angle of front wheels increasing and sideslip angle of rear wheels decreasing, having a tendency to increase understeering; when the lateral force of front and rear wheels is constant and the braking force of rear wheels are larger than front wheel, sideslip angle of rear wheels increasing and sideslip angle of front wheels decreasing, having a tendency to decrease understeering. If only considering the effect of vertical load strength, when braking, the front wheels load increasing and the rear wheels load decreasing, which caused sideslip angle of front wheels decreasing and sideslip angle of rear wheels increasing, then having a tendency to decrease understeering [9]. The decreasing of understeering will make the decreasing of vehicle steering radius, which caused the increasing of centrifugal force. In addition, rainfall will make the decreasing of coefficient of road adhesion, over-speeding will make vehicle centrifugal force increasing. When multiple influence factors have effects on vehicle, driving risk increased dramatically, finally, sideslip happened and endangers driving safety.

\subsection{Evaluation Index of Vehicle Moving Risk}

At present, the evaluation indices of vehicle are lateral displacement ratio, lateral acceleration, etc. Lateral displacement ratio refers the ratio of the distance between vehicles offset trajectory and original trajectory and width of the driveway, obviously, only when vehicles have great sideslip, significantly offset happened, however, the force situation is too complex when vehicles have great sideslip to analyze. Therefore, this paper selected lateral acceleration as the evaluation index of vehicle moving risk. In addition, the change of longitudinal force and vertical load strength will endanger driving safety when braking, therefore, selected a new evaluation index od vehicle moving risk- critical adhesion coefficient-to reflect the effect of longitudinal force and vertical load strength.

Lateral acceleration refers vehicles acceleration along road transverse direction when vehicles turning drive, generally speaking, lateral acceleration unfavorable over $4 \mathrm{~m} / \mathrm{s}^{2}$, the reason is that tire cornering characteristics into obvious nonlinear area when lateral acceleration over $4 \mathrm{~m} / \mathrm{s}^{2}$ which results in the steady-state response characteristics changes significantly, the tire sideslip angle and yawing angular velocity change sharply, so that can no longer maintain a circular motion, the steering radius changed rapidly which endanger driving safety [10].
Critical adhesion coefficient means the ratio of vehicle lateral and vertical forces take maximum value after take absolute value. Selected Critical adhesion coefficient as evaluation index of vehicle moving risk considered speed increased, radius decreased will lead to centrifugal force increased, braking behavior lead to lateral force increased, critical adhesion coefficient can respect the critical situation that lateral force is equal to adhesion, when critical adhesion coefficient less than this value, driving dangerous, otherwise, driving safety. The calculation method is as follows:

$i(z)=\max \left(\left|\frac{F_{Y i}(z)}{F_{Z i}(z)}\right|\right)$

In the equation, $\mu(z)$ means critical adhesion coefficient on different station, $i(i=1,2,3,4)$ respectively means left side of the front tire, right side of rear tire, left side of rear tire, right side of rear tire, $F_{Y i}(z)$ means lateral force on different station, $F_{z i}(z)$ means vertical force on different station.

\section{SIMULATION MODEL}

Famous vehicle dynamics experts Thomas D. Gillespie, Michael Sayers and Steve Hann used artificial intelligence language LISP programmed Carsim software, which can according to simply system definition entered by users to deduce complicated multi-rigid-body system dynamics model and generate corresponding computer program, mainly used to simulated and forecasted the vehicle steering stability and brake performance, ride performance, dynamic property, economical efficiency, Fig. (1) shows Carsim software primary interface operation.

\subsection{Road Model}

This paper studied vehicle driving stability on curved sections which need to analysis curve radius and superelevation impact on the risk of vehicle moving on curved sections, therefore, need to build a suitable road model.

Road parameters can be input in the Additional Data. Selected 3D road, in the next menu of road plane, entering coordinate value under different pile numbers to acquire road horizontal section alignment; in the road profile section models, entering the starting and end point elevation to acquire road profile alignment (this paper did not consider the effect of longitudinal slope, therefore, the starting and ending point elevation is same), in the road cross section models, entering typical cross sections under the left, center, right side of the road elevation to get road transect line.

In addition, due to change of adhesion coefficient can only effect on the maximum static friction force which provided by road, therefore, the adhesion coefficient of value has no effect on study with no skid behaviors, so it can take as a fixed value, refer to Table $\mathbf{1}$ [13], the paper took 0.9.

\subsection{Vehicle Model}

Vehicle model is divided into body parameters and system parameters, body parameters including size, mass and other vehicle information, system parameters are 5 modules including engine, braking, steering, suspension, tire. Table 1 list some important body parameters and system parameters. 


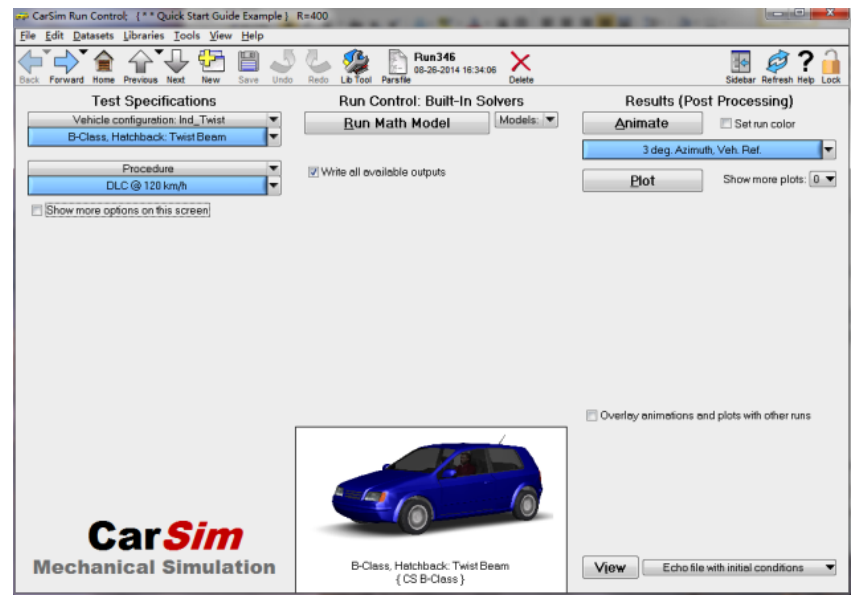

(a). Main interface.

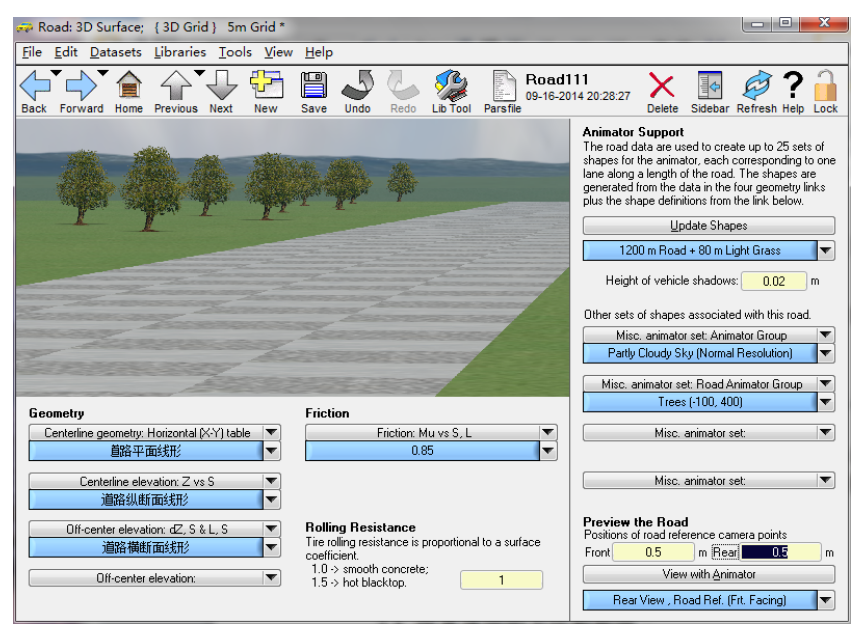

(c). Road parameter settings interface.

Fig. (1). Vehicle model parameter settings interface.

Table 1. Adhesion coefficient under different road conditions.

\begin{tabular}{|c|c|}
\hline Road Condition & Adhesion Coefficient \\
\hline \hline $\begin{array}{c}\text { Dry, Regular maintenance, Construction of } \\
\text { good quality }\end{array}$ & 1.0 \\
\hline Mild polishing & 0.9 \\
\hline Moist & 0.7 \\
\hline Road table macro and micro texture & 0.5 \\
\hline Moist, Polishing & 0.4 \\
\hline Snow & 0.3 \\
\hline Ice & 0.2 \\
\hline
\end{tabular}

\section{PLANE CURVE SECTION RISK INFLUENCE FACTORS ANALYSIS}

The root reason of risk on plane curve section braking behavior is the force provided by road could not be enough to resist the centrifugal force when steering, as shown in Fig. (2) (red vehicle moving safety, blue vehicle sideslip). In theory, all factors maybe have an influence on lateral force, and

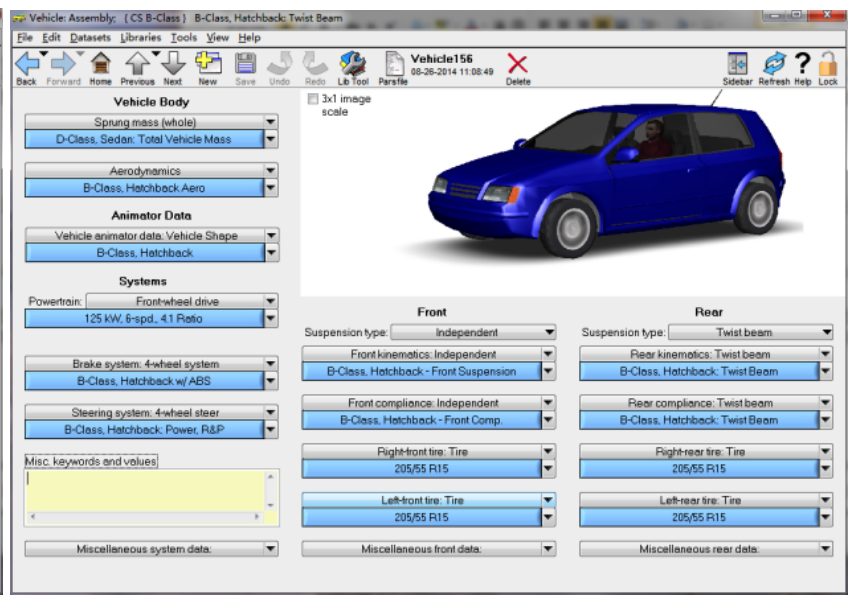

(b)Vehicle parameter settings interface.

\section{Driver Controls}

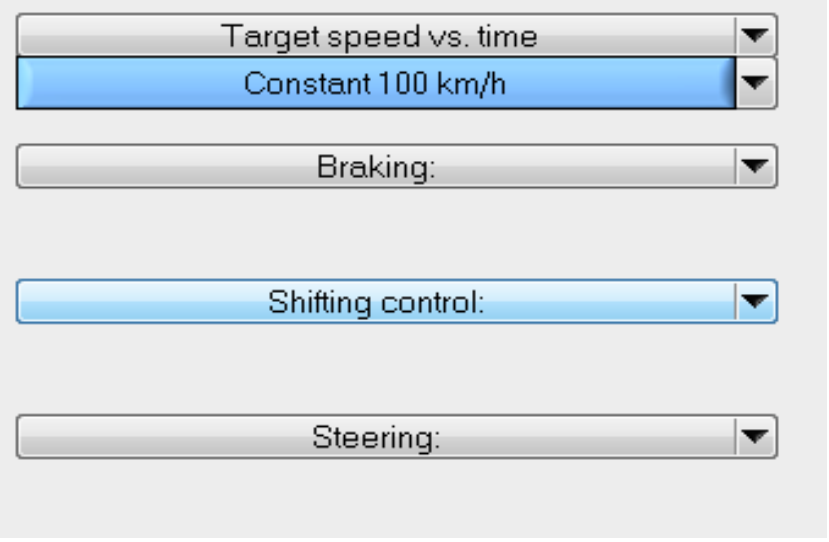

(d) Driver parameter settings interface.

the intensity level of the influence of various factors is different. Therefore, need to analyze whether each factor has an influence on the risk of curve section and the intensity level of each factor.

\subsection{Single Factor Analysis \\ 4.1.1. Simulation Strategy}

Study on whether braking force, circular curve radius, speed and super elevation have impact on the risk of vehicle braking behavior, refer to "Design Specification for Highway Alignment" [14] and "Automotive Engineering Handbook" [15], Table 3 lists simulation strategy.

\subsubsection{Simulation Result Analysis}

In Figs. (3 and 4), $Y$-axis is critical adhesion coefficient $\mu$ and lateral acceleration $a, X$-axis is station, $0-100 \mathrm{~m}$ is straight line segment, $100-225 \mathrm{~m}$ is transition curve, 225 $475 \mathrm{~m}$ is circular curve, $475-600 \mathrm{~m}$ is transition curve, 600$700 \mathrm{~m}$ is straight line segment. Braking behavior way is began to exert barking force when vehicle driving into circular curve of $25 \mathrm{~m}$, the time of exert braking force and release force is $0.5 \mathrm{~s}$, total $1 \mathrm{~s}$. the reason why chose the way to exert braking force is each point on the circular curve radius of 
Table 2. Body parameters and system parameters.

\begin{tabular}{|c|c|}
\hline Vehicle total weight $/ \mathrm{kg}$ & 1530 \\
\hline Vehicle length/mm & 4350 \\
\hline Wheel span/mm & 1481 \\
\hline Wheel base/mm & 2776 \\
\hline Moment of inertia of the vehicle around the X axis/ (kg.m2) & 708 \\
\hline Sliding rate when ABS starts & 0.2 \\
\hline Speed when ABS lose efficacy/(km/h) & 7.5 \\
\hline Kingpin inclination $/^{\circ}$ & 10 \\
\hline Kingpin caster angle ${ }^{\circ}$ & 3 \\
\hline Backward of drag/mm & 8 \\
\hline
\end{tabular}

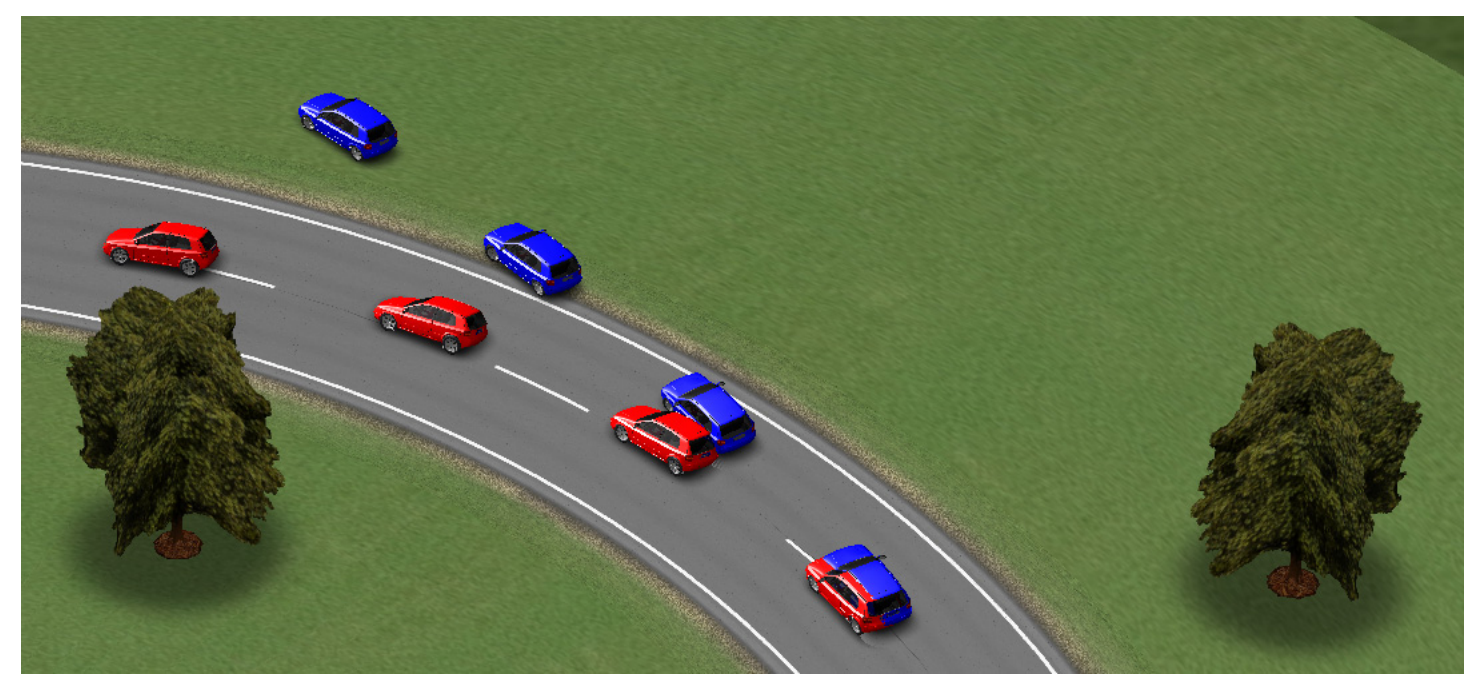

Fig. (2). Scene of sideslip.

Table 3. Simulation strategy considering different road parameters.

\begin{tabular}{|c|c|c|c|c|}
\hline Study Objection & Braking Force F/N & Circular Curve Radius R/m & Speed V/(km/h) & Super Elevation e/\% \\
\hline \hline Braking force & $50,75,100,125$ & 600 & 80 & 6 \\
\hline Circular curve radius & 75 & $250,400,600,800$ & 80 & 6 \\
\hline Speed & 75 & 600 & $60,80,100,120$ & 6 \\
\hline Super elevation & 75 & 600 & 80 & $2,4,6,8$ \\
\hline
\end{tabular}




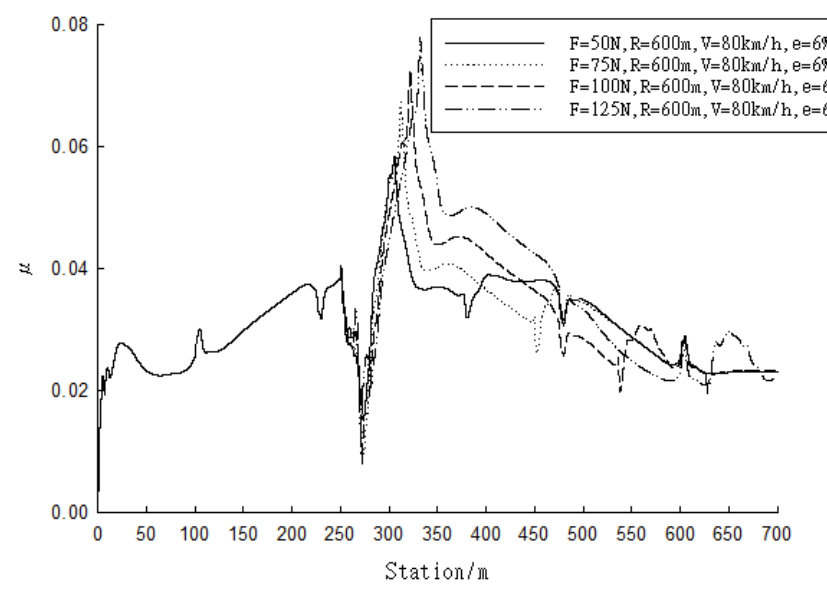

(a)

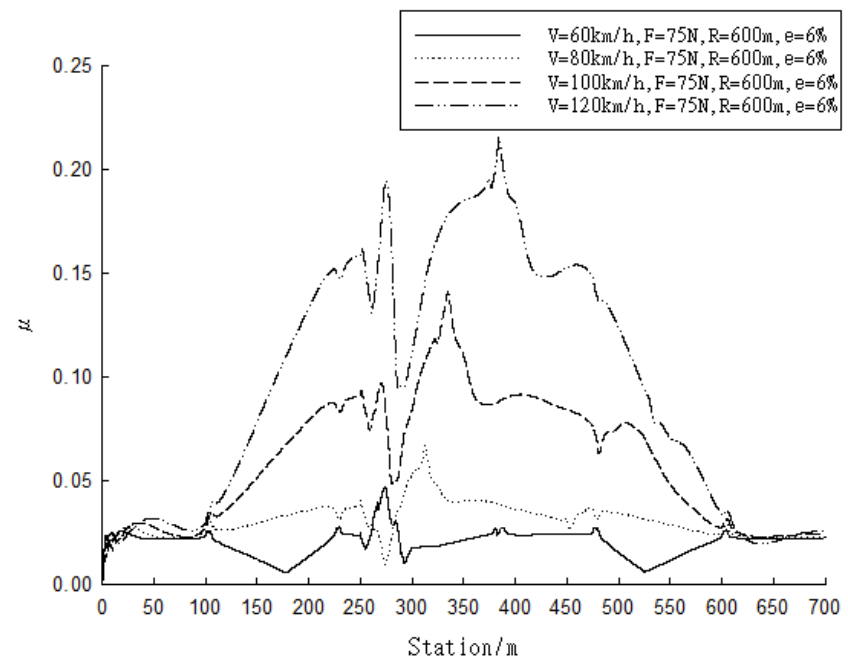

(c)

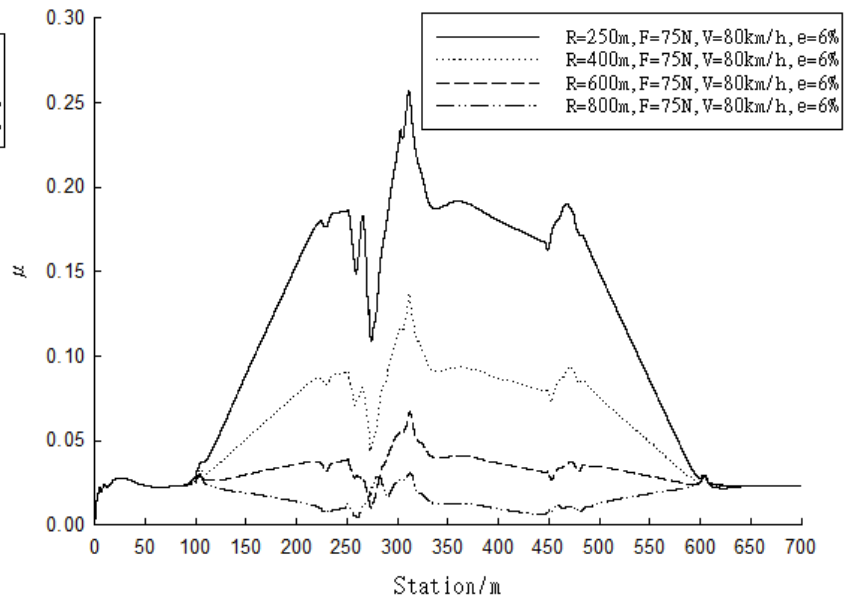

(b)

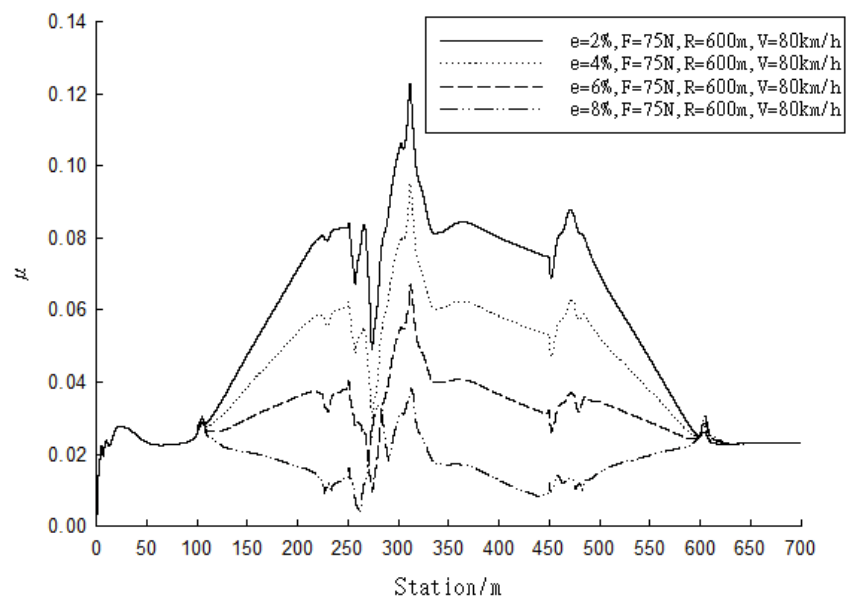

(d)

Fig. (3). Pavement critical adhesion coefficient with different road parameters.

curvature is same, so when other parameters constant, each point of stress distribution is same, therefore, whenever the braking starting, the effect is same, in addition, the driver braking time has a little influence on vehicle braking stability [16], so chose braking time as 1 s has a little impact on study. 4 coordinate graphs in Fig. (3) and (4) reflect critical adhesion coefficient $\mu$ and lateral acceleration $a$ changes under different braking force, circular curve radius, speed, super elevation.

In Figs. (3) and (4), speed from 0 to a constant value need a transition period, so the value of $\mu$ and $a$ has slight fluctuation, $50-100 \mathrm{~m}$, vehicle driving on straight section, the transition has been completed, so value of $\mu$ and $a$ keep stable and tends to $0,100-225 \mathrm{~m}$, vehicle moving on transition road, curve radius decreases gradually which cause centrifugal force increases gradually, the value of $\mu$ and $a$ increased, $225-250 \mathrm{~m}$, vehicle moving on circular curve road, because the radius is constant, so the centrifugal force is constant, the value of $\mu$ and $a$ remain unchanged, $250-475 \mathrm{~m}$, completed a braking operation, the value of $\mu$ and $a$ has fluctuations, 475$600 \mathrm{~m}$, vehicle moving on transition road, curve radius increases gradually which cause centrifugal force decreases gradually, the value of $\mu$ and $a$ decreased, $600-700 \mathrm{~m}$, vehicle driving on straight section, value of $\mu$ and $a$ keep stable and tends to 0. Meanwhile, from Figs. (3) and (4), with the increase of braking force, the value of $\mu$ and $a$ increased gradually, with the decrease of circular curve radius, the value of $\mu$ and $a$ increased gradually, with the increase of speed, the value of $\mu$ and $a$ increased gradually, with the decrease of super elevation, the value of $\mu$ and $a$ increased gradually. Therefore, the value of $\mu$ and $a$ with circular curve radius and super elevation is inversely relationship, with braking force and speed is positive relationship.

\subsection{Multiple Factors Analysis}

\subsubsection{Simulation Strategy}

Here need to analysis of braking force, circular curve radius, speed and super elevation impact on the risk of braking behavior, therefore, refer to "Design Specification for Highway Alignment" [14] and "Automotive Engineering Handbook" [15], respectively selected the value of braking force as $50,75,100,125 \mathrm{~N}$, circular curve radius as $250,400,600$, $800 \mathrm{~m}$, speed as $60,80,100,120 \mathrm{~km} / \mathrm{h}$, super elevation as $2 \%$, $4 \%, 6 \%, 8 \%$. 


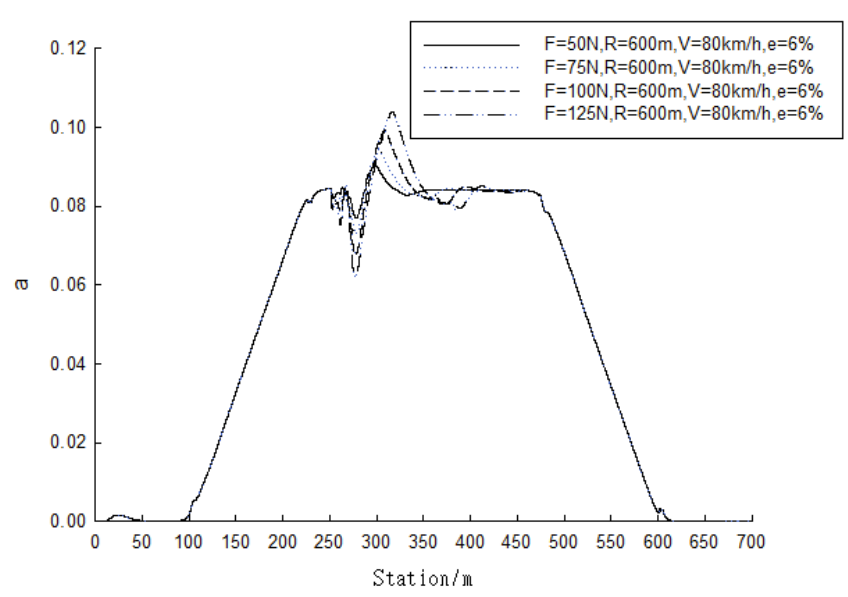

(a)

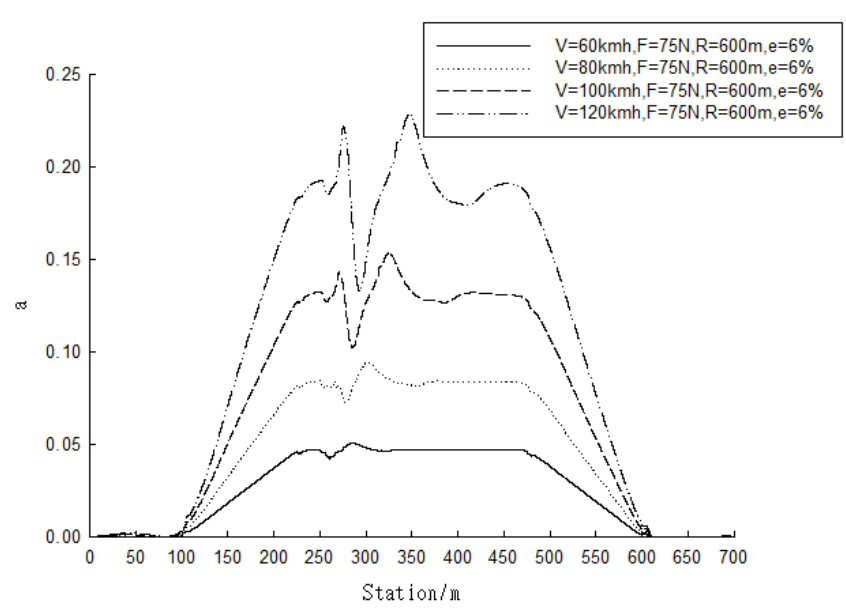

(c)

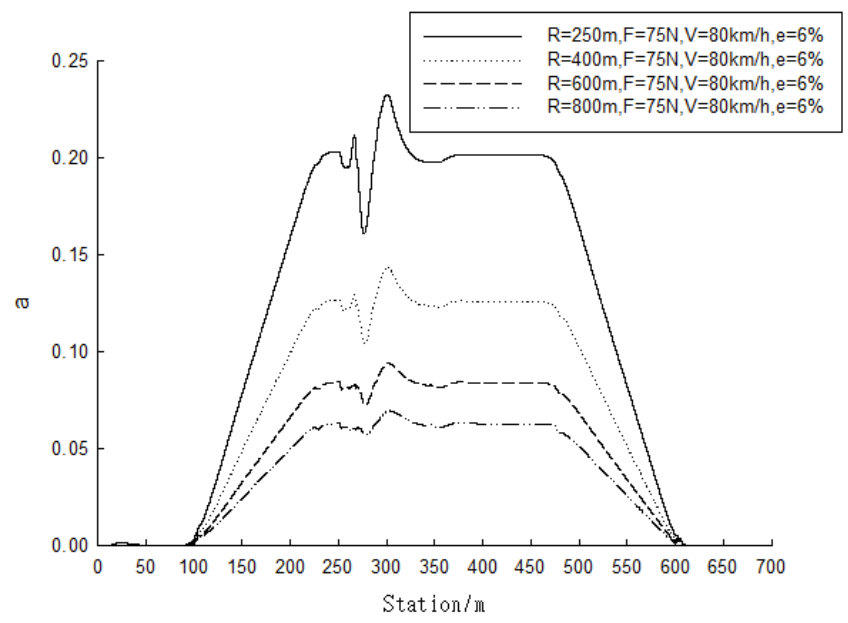

(b)

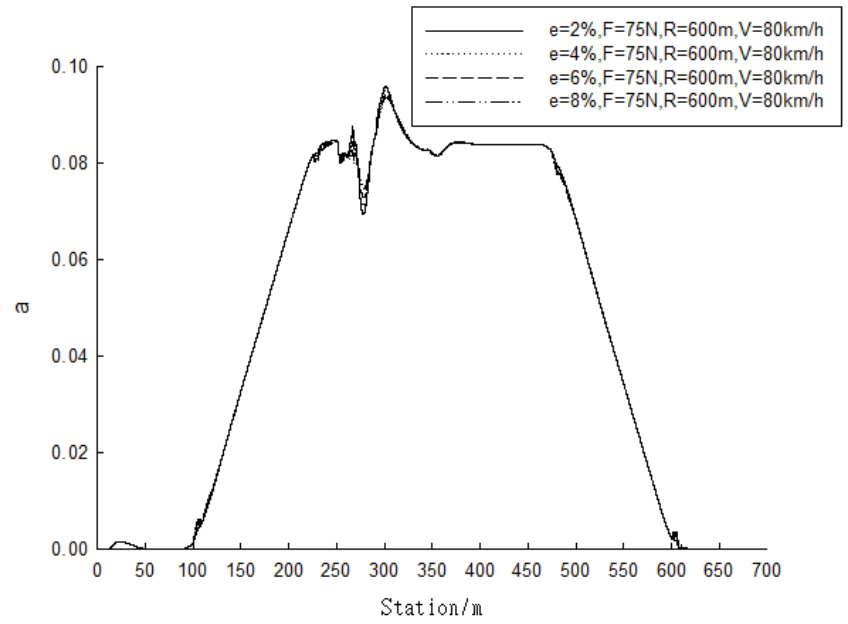

(d)

Fig. (4). Lateral acceleration with different road parameters.

\subsubsection{Orthogonal Test}

This test need to consider 4 factors, each factor has 4 sets of data, to reduce test times, adopt orthogonal experiment to reduce the data set, the orthogonal text table which we chose was $\mathrm{L}_{16}(45)$.

\subsubsection{Results of Simulation Analysis}

Can be seen from Table 4.

(1) $\left.\left.\left.S S_{R}\right\rangle S S_{V}\right\rangle S S_{F}\right\rangle S S_{e}$, the important order of factors (from strong to weak) as circular curve radius, speed, braking force, super elevation.

(2) The $\mathrm{F}$ value of braking force, circular curve radius, speed are above the critical value, however, the $\mathrm{F}$ value of super elevation below the critical value, therefore, braking force, circular curve radius, speed had a significant influence on the risk of plane curve section, super elevation had little influence.

Can be seen from Table 5.

(1) $\left.\left.\left.S S_{V}\right\rangle S S_{R}\right\rangle S S_{F}\right\rangle S S_{e}$, the important order of factors (from strong to weak) as speed, circular curve radius, braking force, super elevation.

(2) The $F$ value of circular curve radius, braking force above the critical value, while the $F$ value of braking force and super elevation below the critical value, therefore, circular curve radius, speed had a significant influence on the risk of plane curve section, braking force and super elevation had little influence.

In conclusion, the difference between results of Tables 4 and $\mathbf{5}$ is the main influencing factor on the risk of driving and the significance of the influence of braking force. The causes of the differences are critical adhesion coefficient and lateral acceleration are two different concepts, in addition, data processed by data, there was a bit error. However, it is known that speed and circular curve radius had significant effect on safety, braking force had common effect, super elevation had little effect.

\section{ESTABLISH EVALUATION MODEL AND RISK ANALYSIS}

\subsection{Evaluation Model}

Rainfall will result in the loss of road adhesion coefficient, in turn, results in the decrease of adhesive force between tire and road, and the greater about rainfall, the smaller about adhesion coefficient, therefore, under the con- 
Table 4. Result of deviation and variance analysis based on the critical adhesion coefficient.

\begin{tabular}{|c|c|c|c|c|c|}
\hline Factors Catalog & Braking Force F/N & Circular Curve Radius R/m & Speed/(km/h) & Super Elevation/\% & Error \\
\hline \hline $\begin{array}{c}\text { Sum of squares of } \\
\text { deviations } S S\end{array}$ & 0.060204 & 0.211119 & 0.203739 & 0.020555 & 0.009705 \\
\hline Number of degrees & 3 & 3 & 3 & 3 & 2.118034 \\
\hline Value of $F$ & 6.203709 & 21.754570 & 20.994080 & $\mathrm{~F}_{0.1}(3,3)=5.36$ & No salience \\
\hline Critical value of $F$ & $\mathrm{~F}_{0.1}(3,3)=5.36$ & $\mathrm{~F}_{0.1}(3,3)=5.36$ & Salience & Salience & \\
\hline Significance & Salience & & & \\
\hline
\end{tabular}

Table 5. Result of deviation and variance analysis based on the lateral acceleration.

\begin{tabular}{|c|c|c|c|c|c|}
\hline Factors Catalog & Braking Force F/N & Circular Curve Radius R/m & Speed/(km/h) & Super Elevation/\% & Error \\
\hline \hline $\begin{array}{c}\text { Sum of squares of } \\
\text { deviations } S S\end{array}$ & 0.032805 & 0.136106 & 0.164453 & 0.008599 & 0.007714 \\
\hline Number of degrees & 3 & 3 & 3 & 3 \\
\hline Value of $F$ & 4.252461 & 17.643252 & 21.317823 & 1.114635 & 3 \\
\hline Critical value of $F$ & $\mathrm{~F}_{0.1}(3,3)=5.36$ & $\mathrm{~F}_{0.1}(3,3)=5.36$ & $\mathrm{~F}_{0.1}(3,3)=5.36$ & $\mathrm{~F}_{0.1}(3,3)=5.36$ & No salience \\
\hline Significance & Salience & Salience & Salience & \\
\hline
\end{tabular}

dition of rainfall, in order to ensure vehicles running in normal curve sections did not sideslip, need big circular curve radius and better road alignment; in addition, when moving on curved sections, increased speed or braking force can lead the increase of centrifugal force, therefore, in order to ensure vehicle did not sideslip on curve section, need to control speed and braking force within a certain range.

In view of this, need to study the effect of rainfall to road friction coefficient and get the model, at the same time, need to analyze the effect of various factors to the value of $\mu$ and $a$ and establish a model, which used to analysis the risk of moving on curved sections under the condition of rainfall and over-speeding.

\subsubsection{Adhesion Coefficient and Rainfall Model}

Rainfall will not only adds the difficulties to driving, also are more likely to cause traffic accidents, the reason is rainfall results in road surface covering a layer of water film, vehicles moving on the road, caused by the water film lubrication, adhesion coefficient decreased significantly, which lead to uncontrollable braking, ineffective steering, vehicle easy to sideslip, deviating from the normal driving direction. The relationship between road adhesion coefficient and rainfall capacity, has been the main content of the study of traffic accident which under the bad weather, Chinese scholar JI Tianjian used experimental data regression analysis the relationship between water film thickness and rainfall intensity, the relationship between adhesion coefficient under different vehicle models and water film thickness, merge two equations can get the relationship between adhesion coefficient and rainfall intensity, the calculation method is as shows in formula (2). $\varnothing=0.9458-0.0057 v-0.0007 l^{0.6134} \times a^{-0.3133} \times q^{1.4483}\left(r^{2}=0.88\right)$

In the formula, $\psi, v, l, \alpha, q$ respectively indicates adhesion coefficient, speed $(\mathrm{km} / \mathrm{h})$, the length of slope $(\mathrm{m})$, synthetic cross slope, rainfall intensity $(\mathrm{mm} / \mathrm{min})$.

\subsubsection{Value of $\mu$, a and Road Factors Model}

According to the analysis in third quarter, super elevation has little effect on the value of $\mu$ and $a$ which can ignored, therefore, value of $\mu, a$ and road factors model can be expressed as:

$\left\{\begin{array}{l}\grave{i}=f_{1}(R, v, F) \\ a=f_{2}(R, v, F)\end{array}\right.$

In the formula, $R, v, F$ respectively indicates circular curve radius $(\mathrm{m})$, speed $(\mathrm{km} / \mathrm{h})$, braking force $(\mathrm{N})$.

Put the 16 groups of data which analyzed in multiplefactors into Matlab, regression analysis the value of $\mu$ and a, got specific regression model:

$\left\{\begin{array}{l}i=\frac{0.012268 v^{2}}{R}+0.000745 F-0.098270\left(r^{2}=0.96\right) \\ a=\frac{0.102900 v^{2}}{R}+0.003704 F-0.493920\left(r^{2}=0.93\right)\end{array}\right.$

\subsection{Analysis Risk of Curved Sections Braking Behavior under the Condition of Rainfall, Over-Speeding}

Used formula 4, can analysis the risk of curved sections braking behavior under the condition of rainfall, overspeeding, refer to "Design Specification for Highway 
Table 6. Risk analysis of vehicle moving on curved sections.

\begin{tabular}{|c|c|c|c|c|c|c|c|c|c|c|}
\hline $\begin{array}{c}\text { Slope Length } \\
\quad l / \mathrm{m}\end{array}$ & $\begin{array}{c}\text { Super Eleva- } \\
\text { tion } e / \%\end{array}$ & $\begin{array}{c}\text { Braking } \\
\text { Force } F / \mathbf{N}\end{array}$ & $\begin{array}{c}\text { Rainfall } \\
\text { Intensity } \boldsymbol{q} \\
/(\mathbf{m m} / \mathbf{m i n})\end{array}$ & $\begin{array}{c}\text { Circular } \\
\text { Curve Ra- } \\
\text { dius } R / \mathbf{m}\end{array}$ & $\begin{array}{l}\text { Driving } \\
\text { Speed } V \\
/(\mathbf{k m} / \mathbf{h})\end{array}$ & $\begin{array}{l}\text { Road Adhe- } \\
\text { sion Coeffi- } \\
\text { cient } \psi\end{array}$ & $\begin{array}{c}\text { Critical Ad- } \\
\text { hesion Coef- } \\
\text { ficient } \mu\end{array}$ & $\begin{array}{c}\text { Lateral Ac- } \\
\text { celeration } \\
a_{t} /\left(\mathbf{m} / \mathbf{s}^{2}\right)\end{array}$ & $\begin{array}{c}\text { Lateral Ac- } \\
\text { celeration } \\
\text { Threshold } \\
a /\left(\mathbf{m} / \mathbf{s}^{2}\right)\end{array}$ & Driving Risk \\
\hline \multirow{8}{*}{11.25} & \multirow{8}{*}{5} & \multirow{8}{*}{50} & \multirow{4}{*}{0} & \multirow{2}{*}{250} & 80 & 0.490 & 0.250 & 2.325 & 4 & Safety \\
\hline & & & & & 96 & 0.399 & 0.388 & 3.485 & 4 & Safety \\
\hline & & & & \multirow{2}{*}{400} & 80 & 0.490 & 0.134 & 1.338 & 4 & Safety \\
\hline & & & & & 96 & 0.399 & 0.219 & 2.062 & 4 & Safety \\
\hline & & & \multirow{4}{*}{1.5} & \multirow[b]{2}{*}{250} & 80 & 0.476 & 0.250 & 2.325 & 4 & Safety \\
\hline & & & & & 96 & 0.384 & 0.388 & 3.485 & 4 & $\begin{array}{l}\text { Critical } \\
\text { balance }\end{array}$ \\
\hline & & & & \multirow{2}{*}{400} & 80 & 0.476 & 0.134 & 1.338 & 4 & Safety \\
\hline & & & & & 96 & 0.384 & 0.219 & 2.062 & 4 & Safety \\
\hline \multirow{8}{*}{11.25} & \multirow{8}{*}{5} & \multirow{8}{*}{350} & \multirow{4}{*}{0} & \multirow{2}{*}{250} & 80 & 0.490 & 0.475 & 3.436 & 4 & Safety \\
\hline & & & & & 96 & 0.399 & 0.611 & 4.595 & 4 & Danger \\
\hline & & & & \multirow{2}{*}{400} & 80 & 0.490 & 0.357 & 2.448 & 4 & Safety \\
\hline & & & & & 96 & 0.399 & 0.443 & 3.173 & 4 & Danger \\
\hline & & & \multirow{4}{*}{1.5} & \multirow[t]{2}{*}{250} & 80 & 0.476 & 0.475 & 3.436 & 4 & $\begin{array}{c}\text { Critical bal- } \\
\text { ance }\end{array}$ \\
\hline & & & & & 96 & 0.384 & 0.611 & 4.595 & 4 & Danger \\
\hline & & & & \multirow{2}{*}{400} & 80 & 0.476 & 0.357 & 2.448 & 4 & Safety \\
\hline & & & & & 96 & 0.384 & 0.443 & 3.173 & 4 & Danger \\
\hline
\end{tabular}

Alignment" (14) and "Automotive Engineering Handbook" (15), under the condition of design speed as $80 \mathrm{~km} / \mathrm{h}$, respectively chose braking force as $50 \mathrm{~N}$ (general braking) and $350 \mathrm{~N}$ (emergency braking), length of slope as $11.25 \mathrm{~m}$ (regardless of the longitudinal slope, slope length is half of the roadbed width), circular curve radius as $250 \mathrm{~m}$ and $400 \mathrm{~m}$, speed as $80 \mathrm{~km} / \mathrm{h}$ and $96 \mathrm{~km} / \mathrm{h}$ ( $20 \%$ over-speeding), super elevation as $5 \%$, rainfall intensity as 0 and $1.5 \mathrm{~mm} / \mathrm{min}$ (rainstorm), finally, got the risk of curved sections braking behavior under the condition of rainfall, over-speeding, shows in Table 6.

\section{See from Table 6.}

(1) When taking general braking behavior, even if road alignment is poor, speed faster and with bad weather condition, the vehicles will not sideslip. For example, when the braking force as $50 \mathrm{~N}$, rainfall intensity as $1.5 \mathrm{~mm} / \mathrm{min}$, circular curve radius as $250 \mathrm{~m}$, speed as $96 \mathrm{~km} / \mathrm{h}$, the value of $\psi$ is 0.384 the value of $\mu$ is 0.388 the value of a is $3.485 \mathrm{~m} / \mathrm{s}^{2}$ the value of $a_{t}$ is $4 \mathrm{~m} / \mathrm{s}^{2}$ in the limit cases, only when considering from adhesion coefficient, just at the critical state of equilibrium, when considering from lateral acceleration, at a safe state.

(2) When taking emergency braking behavior, if overspeeding, no matter road alignment and weather is good or not, it is likely to sideslip. For example, when the braking force as $350 \mathrm{~N}$, speed as $96 \mathrm{~km} / \mathrm{h}$, rainfall intensity as 0 and circular curve radius as $250 \mathrm{~m}$ or $1.5 \mathrm{~mm} / \mathrm{min}$ and $250 \mathrm{~m}$ or $1.5 \mathrm{~mm} / \mathrm{min}$ and $400 \mathrm{~m}$, at this point, although lateral acceleration in a safe state, but $\psi$ less than $\mu$, may occur sideslip.

(3) When the rainfall intensity exceeds $1.5 \mathrm{~mm} / \mathrm{min}$, taking emergency braking behavior, even if not over-speeding and limit-state criterion requirement is meet, vehicles are easy to sideslip. For example, when the braking force as $350 \mathrm{~N}$, rainfall intensity as $1.5 \mathrm{~mm} / \mathrm{min}$, circular curve radius as $250 \mathrm{~m}$, speed as $80 \mathrm{~km} / \mathrm{h}$, at this point, the value of $\psi$ is 0.476 , the value of $\mu$ is 0.475 , in the critical state of equilibrium, sideslip may occur.

(4) Consideration from adhesion coefficient, driving has risk, while consideration from lateral acceleration, driving may not has risk; consideration from lateral acceleration, driving has risk, consideration from adhesion coefficient, driving also has risk. For example, when braking force as $350 \mathrm{~N}$, rainfall intensity as 0 , circular curve radius as $400 \mathrm{~m}$, speed as $96 \mathrm{~km} / \mathrm{h}$, the value of $\psi$ as 0.399 , the value of $\mu$ as 0.443 , the value of as a $3.173 \mathrm{~m} / \mathrm{s}^{2}$, the value of as $a_{t}$ as $4 \mathrm{~m} / \mathrm{s}^{2}$, at this point, consideration from adhesion coefficient, will sideslip, however, when consideration from lateral acceleration, driving safety. Therefore, when evaluation vehicles driving risk, meet the requirements of adhesion coefficient, will also meet the requirements of lateral acceleration. 


\section{CONCLUSION}

(1) Based on vehicle dynamics, used Carsim software, simulation of vehicle with different speed under the condition of all road factors, and considered the effect of braking force, found the braking force, circular curve radius, speed and super elevation impact on the risk of vehicle moving on curved sections.

(2) Orthogonal design is used to reduce the test data, effect factors and the efficiency are analyzed based on the knowledge of mathematical statistics, the results show that speed and radius of horizontal curve have significant effects on the risk of driving, braking effect is moderate, superelevation has few effect.

(3) Consider influence factors, used Matlab software, regression analysis the data got by Carsim software simulation, established the model between adhesion coefficient and influenced factors and the model between lateral acceleration and impact factors.

(4) When taking general braking behavior, even with the condition of poor road alignment, faster speed and bad weather, vehicle will not sideslip; when taking emergency braking behavior, if over-speeding, no matter road alignment and weather is good or not, it is possible to sideslip.

(5) When evaluation the risk of vehicle, meet the requirements of adhesion coefficient, also meet the requirements of lateral acceleration.

(6) In this paper, the vehicle model single, and no consider the different braking force distribution on the risk of vehicle, next stage, should consider the braking force distribution for get more general conclusion.

\section{CONFLICT OF INTEREST}

The authors confirm that this article content has no conflict of interest.

\section{ACKNOWLEDGEMENTS}

The authors would like to acknowledge National Science and Technology Support Plan (2014BAG05B01), The Ministry of Education of Doctoral Fund Project of the New Teacher (20120205120013), The Ministry of Transport Ap- plied Basic Research Project (2014319812170), The Central University Basic Business Expenses Special Funds for Scientific Research Projects (2013G2211005 \& CHD2011JC048). Their supports are gratefully acknowledged.

\section{REFERENCES}

[1] J. H. Gao, and W. Wang, "Highway alignment design". The Yellow River water conservancy press., vol. 9, 2005 (In Chinese)

[2] J. Andrey, and S. Yagar, " $A$ temporal analysis of rain-related crash risk”, Accident Analysis \& Prevention, vol. 25, no. 4, pp. 465-472, 1993.

[3] Z. C. Su, "Study on vehicle dynamic stability control on steering and braking maneuvers", Chongqing University, 2007. (In Chinese)

[4] Turner-Fairbank Highway Research Center. "Evaluation of Design Consistency Methods for Two-Lane Rural Highways", FHWARD-99-174. Federal Highway Administration, Washington DC, 1999. (In Chinese)

[5] C. V. Zegeer, J. R. Stewart, and F. M. Council, "Safety Effects of Geometric Improvements on Horizontal Curves", Transportation Research Board, Washington D C, 1991.

[6] D. O. Cinneide, "The relationship between geometric design standards and safety" //AASHTO, International Symposium on Highway Geometric Design Practices, 1998.

[7] C. Zegeer, R. Stewart, and D. Reinfurt, "Cost effective geometric improvements for safety upgrading of horizontal curves", FHWARD-90-021. FWHA, Washington D C, 1990.

[8] J. Xu, "Effects of change in road geometry design on vehicle driving dynamics", Southwest Jiaotong University, 2006. (In Chinese)

[9] "Automotive engineering handbook" editorial board, "Automotive engineering manual, base paper”, People's Traffic Press, 2001. (In Chinese)

[10] Z. S. Yu, "Automobile theory", Mechanical Industry Press, 2009. (In Chinese)

[11] S. Dupuy, A. Egges, V. Legendre, and P. Nugues, "Generating a 3D simulation of a car accident from a written description in natural language: the CarSim system", Proceedings of the workshop on Temporal and spatial information processing, 2001.

[12] L. Xiong, C. Chen, and Y. Fen, "Modeling of distributed drive electric vehicle based on co-simulation of Carsim/Simulink", Journal of System Simulation, 2014. (In Chinese)

[13] K. K. You, L. Sun, W. J. Gu, "Reliability design theory and method of highway horizontal curve radius", Journal of Traffic and Transportation Engineering, 2012. (In Chinese)

[14] JTG D20-2006, Highway route design specification.

[15] "Automotive engineering handbook" editorial board. "Automotive engineering manual, design paper”, People's Traffic Press, 2001. (In Chinese)

[16] J. Xu. "Quantitative analysis of the influence of drivers' behavior on braking security. Human ergonomics", 2007. (In Chinese)

[17] T. J. Ji, "The influence of rainfall on the tire and road adhesion coefficient", Southeast University, 2004. (In Chinese). 